\title{
Study on chemical constituents of different species of Dendrobium
}

\author{
Zhenghui Wan ${ }^{1, *}$, Qingjun Huang ${ }^{1}$ \\ ${ }^{1}$ School of Ecology Technology and Engineering, 201418 Shanghai Institute of Technology, Shanghai China
}

\begin{abstract}
The objective of this study is to determine the erianin of 10 species of Dendrobium by high performance liquid chromatography (HPLC). Processing techniques were washed, dried and ground. The research method for determining the content of erianin was adopted from Chinese Pharmacopoeia 2015 Edition. Acetonitrile- $0.05 \%$ phosphoric acid (37:63) was used as mobile phase. The samples were separated on Sharpsil-TC18 column $\left(4.6^{*} 150 \mathrm{~mm} ; 5 \mu \mathrm{m}\right)$ at a flow rate of $1.2 \mathrm{~mL} / \mathrm{min}$ and detected at $230 \mathrm{~nm}$, and the column temperature was kept at $30^{\circ} \mathrm{C}$. The injection volume was $20 \mu \mathrm{L}$. The number of theoretical plates was not less than 6000 according to the chromatographic peak of erianin. The result of the study showed erianin was detected only in Dendrobium chrysotoxum among the 10 species of Dendrobium. and the content was $0.098 \%$. Conclusion: The content of erianin in Dendrobium chrysotoxum met the specification of no less than $0.03 \%$ in the 2015 edition of Chinese Pharmacopoeia.
\end{abstract}

\section{INTRODUCTION}

Dendrobium is one of the richest genus of Orchidaceae with more than 1100 species widely distributed in Asian, Europe and Australia. There are 74 species and 2 variations of Dendrobium found in China which are mainly distributed in Yunnan, Sichuan and Guangxi provinces ${ }^{[1]}$. Dendrobium is one of the most well-known orchids in global horticultural trade due to its beautiful flowers and ideal characteristics as houseplants. Moreover, Dendrobium has been used as traditional herbal in many Asian countries for a long time. Thus, wild Dendrobium resources have been over-excavated, making wild Dendrobium endangered in recent years.

As a traditional medicinal and ornamental plant in China, Dendrobium has a long history of application. The stems of Dendrobium have been used as medicine for a thousand years. In recent decades, more than 100 compounds have been found and identified from the medical parts of Dendrobium, which include alkaloids, polysaccharide, phenols, flavonoids, amino acids, tanning, etc ${ }^{[2]}$. Modern pharmacological studies show that Dendrobium has the functions of anti-tumor, immune regulation, anti-oxidation, anti-angiogenesis, anti-inflammatory, and relieving diabetes ${ }^{[3]}$. At present, the research on the chemical components of Dendrobium is mainly focused on the polysaccharides and alkaloids, but the research on other chemical components is relatively less. Erianin(2-Methoxy-5-[2-(3,4,5trimethoxyphenyl) ethyl] benzolol) is a low molecular weight natural compound isolated from Dendrobium chrysotoxum, with a relative molecular weight of 318.36 . As a bibenzyl compound, erianin has many pharmacological activities. Sun et al. have found that erianin can significantly inhibit the proliferation of human breast cancer T47D cells, activate immune escape signal pathway to induce apoptosis of T47D cells, inhibit the expression of cyclin dependent kinase (CDKs), and lead to cell cycle arrest, and erianin does not affect the proliferation of normal breast epithelial mcf10a cells [4]. Deng et al found that erianin can induce apoptosis of human lung cancer A549 cells in vitro [5]. Su et al. showed that erianin could block the cell cycle of human hepatoma cell line Huh7 at G2 / m, thus inhibiting its proliferation [6]. Erianin can inhibit cancer through multi-mechanism and multi-layer surface, it has great application prospect in clinical treatment [7]. In this study, the content of erianin in 22 species of Dendrobium was detected by high performance liquid chromatography in order to provide the relevant basis for the quality comparison of different species of Dendrobium, and provide the relevant reference value for the further study of Dendrobium.

\section{MATERIALS AND METHODS}

\subsection{Instrumentation and reagents}

The tools used in this study were analytical scales (analytical balance) with accuracy of $0.01 \mathrm{mg}$, electricity heat drum, beaker cups, pipette, Agilent 1100 high performance liquid chromatograph(diode-array detector), ultrasonic cleaner, conical flask with cover, pulverizer, standard sieve, amber laboratory bottle, label paper, Sharpsil-T C18 column $\left(4.6^{*} 150 \mathrm{~mm} ; 5 \mu \mathrm{m}\right), 0.45 \mu \mathrm{m}$ pore size (Millipore) membrane filter, vacuum pump.

\footnotetext{
* Corresponding author: wanzhenghuiya@163.com
} 
The material that will be used in this research is 10 species of Dendrobium collected from Yunnan and Sichuan. All the Dendrobium stems are healthy and disease-free fresh. These 10 species of Dendrobium are Dendrobium thyrsiflorum, Dendrobium williamsonii, Dendrobium pendulum, Dendrobium nobile, Dendrobium linawianum, Dendrobium densiflorum, Dendrobium wardianum, Dendrobium chrysanthum, Dendrobium ellipsophyllum, Dendrobium chrysotoxum. The chemical used for extracting erianin is methanol (grade HPLC). Erianin (purity $\geq 98.0 \%$ HPLC) was purchased from Shanghai yuanye Bio-Technology Company. The chemicals used for mobile phase are acetonitrile (grade HPLC), phosphoric acid (grade AR), and pure water which was prepared using purified water from a Mili-Q filtration system.

\subsection{Method}

\subsubsection{Chromatographic condition}

The mobile phase was acetonitrile- $0.05 \%$ - phosphoric acid (37:63). The mobile phase was filtered through a $0.45 \mu \mathrm{m}$ pore size (Millipore) membrane filter. The separation was carried out on a Sharpsil-T C18 column $\left(4.6^{*} 150 \mathrm{~mm} ; 5 \mu \mathrm{m}\right)$. The temperature of the column was kept at $30^{\circ} \mathrm{C}$ and the injection volume was $20 \mu \mathrm{l}$. The total run time was set at $30 \mathrm{~min}$. The flow rate was set at $1.2 \mathrm{ml} / \mathrm{min}$ and the detection wavelength was set at $230 \mathrm{~nm}$. Under the above conditions, the number of theoretical plates is 6542 which is better than the regulation in Chinese Pharmacopoeia.

\subsubsection{Preparation of standard solution}

A standard stock solution of erianin was prepared by dissolving appropriate erianin in $50 \mathrm{ml}$ methanol to make a standard solution with a concentration of $15.3 \mu \mathrm{g} / \mathrm{ml}$.

\subsubsection{Preparation of sample solution}

Take the healthy and disease-free fresh stem segments of this 10 species of Dendrobium, and remove the leaves and leaf sheaths. Dry these stems in the electricity heat drum, after that, crush the dry stems, sift them through No. 3 sieve. Accurately take $1 \mathrm{~g}$ powders, put them in a conical flask with a stopper, add $50 \mathrm{ml}$ methanol in it. After soaking them for $20 \mathrm{~min}$, then exposed the solution to ultrasound treatment $(250 \mathrm{~W}, 40 \mathrm{kHz})$ for $45 \mathrm{~min}$. Make up the weight loss with methanol, then filtered through a $0.45 \mu \mathrm{m}$ pore size membrane filter, take the subsequent filtrate as sample solution.

\subsubsection{System suitability test}

Accurately weigh $9.3 \mathrm{mg}$ (M1) of standard erianin, add appropriate methanol to prepare standard solution (std1) with a concentration of $13.95 \mu \mathrm{g} / \mathrm{ml}$; precisely weigh $10.2 \mathrm{mg}$ (M2) of standard erianin, add appropriate methanol to prepare standard solution $(\operatorname{std} 2)$ with a concentration of $15.3 \mu \mathrm{g} / \mathrm{ml}$. Inject std1 6 times continuously, as shown in Table 1; inject std2 9 times continuously, as shown in Table 2 . The recovery rate of standard solution $=((\mathrm{S} 2 / \mathrm{S} 1) * \mathrm{M} 1) / \mathrm{m} 2 * 100 \%$, the average recovery rate of standard solution is $97.4 \%$, which indicating that the system has good suitability.

Table1. STD1 continuous injection

\begin{tabular}{cccc}
\hline $\begin{array}{c}\text { Number of } \\
\text { injection }\end{array}$ & Peak area & $\begin{array}{c}\text { Mean peak } \\
\text { area(S1) }\end{array}$ & RSD \\
\hline 1 & 323 & & \\
2 & 318.2 & & \\
3 & 327.7 & & \\
4 & 327.3 & 325.8 & $1.5 \%$ \\
5 & 325.6 & & \\
6 & 333.1 & & \\
\hline
\end{tabular}

Table2. STD2 continuous injection

\begin{tabular}{cccc}
\hline $\begin{array}{c}\text { Number of } \\
\text { injection }\end{array}$ & Peak area & $\begin{array}{c}\text { Mean peak } \\
\text { area(S2) }\end{array}$ & RSD \\
\hline 1 & 347.6 & & \\
2 & 348.2 & & \\
3 & 345.4 & & \\
4 & 333.1 & 348.2 & \\
5 & 352.7 & & \\
6 & 357 & & \\
7 & 353.5 & & \\
8 & 351.6 & & \\
9 & 345 & & \\
\hline
\end{tabular}

\subsubsection{Determination of samples}

Inject $20 \mu \mathrm{l}$ standard solution and sample solution precisely and inject them into the high performance liquid chromatograph for determination, record the peak area. Calculate the content of erianin in the sample by external standard method.

\section{RESULTS AND DISSCUSION}

Among the 10 species of Dendrobium detected, erianin was detected only in Dendrobium chrysotoxum. The HPLC chromatograms have been shown in Fig1 and

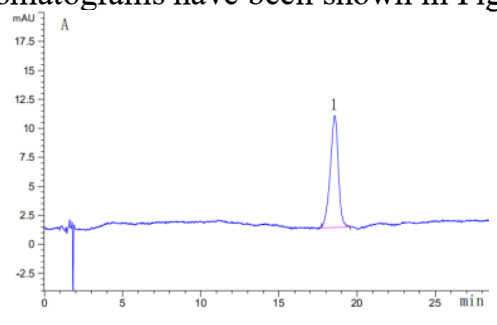

Fig1. Erianin standard; 1 Erianin peak

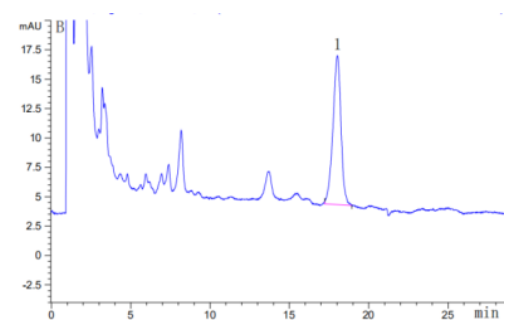

Fig2. Dendrobium chrysotoxum; 1 Erianin peak 
Fig2. It is shown in Fig1 that retention time of erianin peak is about $18.5 \mathrm{~min}$. The content of erianin in these 10 species of Dendrobium is shown in Table3. Content of erianin in Dendrobium chrysotoxum is $0.098 \%$ on the dried basis, which met the specification of no less than $0.03 \%$ in the 2015 edition of Chinese Pharmacopoeia.

Table3. Content of erianin in 10 species of Dendrobium

\begin{tabular}{lcccccc}
\hline \multicolumn{1}{c}{ Variety } & $\begin{array}{c}\text { Sample } \\
\text { weight }(\mathrm{g})\end{array}$ & $\begin{array}{c}\text { Methanol } \\
\text { volume }(\mathrm{ml})\end{array}$ & $\begin{array}{c}\text { Sample } \\
\text { peak area }\end{array}$ & $\begin{array}{c}\text { Standard } \\
\text { peak area }\end{array}$ & $\begin{array}{c}\text { Standard } \\
\text { concentration }(\mu \mathrm{g} / \mathrm{ml})\end{array}$ & Content $(\%)$ \\
\hline D. thyrsiflorum & 1.0075 & 50 & 0 & 348.2 & 15.3 & 0.00 \\
D. williamsonii & 1.0031 & 50 & 0 & 348.2 & 15.3 & 0.00 \\
D. pendulum & 1.0047 & 50 & 0 & 348.2 & 15.3 & 0.00 \\
D. nobile & 1.0002 & 50 & 0 & 348.2 & 15.3 & 0.00 \\
D. linawianum & 1.0030 & 50 & 0 & 348.2 & 15.3 & 0.00 \\
D. densiflorum & 1.0067 & 50 & 0 & 348.2 & 15.3 & 0.00 \\
D. wardianum & 1.0015 & 50 & 0 & 348.2 & 15.3 & 0.00 \\
D. chrysanthum & 1.0034 & 50 & 0 & 348.2 & 15.3 & 0.00 \\
D.ellipsophyllum & 0.9249 & 50 & 0 & 348.2 & 15.3 & 0.00 \\
D. chrysotoxum & 1.0035 & 50 & 447.2 & 348.2 & & 0.098 \\
\hline
\end{tabular}

In this study, no erianin was found in the Dendrobium nobile, which was different from the study of $\mathrm{Xu}$ [8]. And the content of Dendrobium chrysotoxum in this study is significantly different from the study of Xia [9]. What caused these situations may have many explanations. Such as the different growth age, cultivation ways and origin $[10,11]$. Generally speaking, the content of erianin in Dendrobium is significantly affected by many factors. Thus it can be seen that the content of erianin in Dendrobium is controlled by the interaction between genes and environment.

\section{CONCLUSION}

Over the years, many scholars at home and abroad have studied the chemical constituents of Dendrobium, from which alkaloids, polysaccharides, phenanthraquinones, benzenes, amino acid, fluorenones, coumarins, steroids, triterpenoids glycosides and volatile oil have been isolated and identified, and their pharmacological activities have been widely studied. $\mathrm{Xu}$ et al found that only 11 species of Dendrobium are involved in the antitumour study, and there is little research on the antitumour of benzenes compounds extracted from Dendrobium, meanwhile there is no systematic and indepth study [12]. As a natural product isolated from Dendrobium chrysotoxum, erianin has great potential in clinical application of anti-tumour therapy. A large number of studies have shown that erianin can inhibit the occurrence and development of tumour through multiple mechanisms and levels [13].

The suggestions for further research work are as follows. First, more Dendrobium species should be detected and compared. Second, the study of anticancer components in Dendrobium should not only be limited to the erianin. Third, as one of the main components in Dendrobium, bibenzyl may has similar pharmacological effect with erianin. Liquid chromatograph mass spectrometer (LC-MS) method can be used for structural analysis and identification before further research. Fourthly, in order to get high quality Dendrobium, cultivation ways of Dendrobium should be improved.

\section{REFERENCE}

1. Z J Li, Y C Wang, B Han et al. Research progress on constituents of alkaloids in plants from Dendrobium $S w$ [J]. Chinese Traditional and Herbal Drugs. 2018, 50(13):3246-3254

2. X Q Zhang, T M Zhao, J Liu et al. Advances in chemical compounds and pharmacological effects of Dendrobii Caulis [J]. Chinese Traditional and Herbal Drugs 2019, 49(13) :3174-3182

3. X Y Qu, Y X Wu, J L Zhang. Research Advances on Chemical Constituents and Pharmacological Activities of Dendrobium [J]. Tianjin Agricultural Science. 2015, 21(4) :19-23.

4. J Sun, X Fu, Y Wang et al. Erianin inhibits the proliferation of T47D cells by inhibting cell cycles, inducing apoptosis and suppressing migration [J]. Am J Transl Res , 2016, 8(7) :3077-3086

5. T X Deng, M L Wang, W J Wen et al. Erianin induces apoptosis of human lung cancer A549cells via ROS/p38 MAPK pathway [J]. Chinese Journal of Pathophysiology, 2019, 35(8) :1557-1462

6. P Su, J Wang, J X An et al. Inhibitory Effect of Erianin on Hepatocellular Carcinoma (HCC) Huh7 Cells [J]. Chinese Journal of Applied \& Environmental Biology, 2011, 17(50) :662-665.

7. Z Li, Y O Cao, L J Xiao et al. Research Progress of Antitumor Effect of Erianin and its Derivatives [J]. Chinese Traditional Patent Medicine, 2019, 41(10) :2442-2444

8. L Xu, J R Wang, L Guo et al. Chemical constituents from Dendrobium nobile [J]. Chinese Traditional Patent Medicine, 2018, 40(5) :1110-1112

9. K Z Xia, Z Wang, L Z Chen. Study on the Determination of Antitumor Component Erianin in Dendrobium chrysotoxum [J]. Chinese Journal of Modern Applied Pharmacy, 2008, 25(1) :63-66

10. H Yang, Z P Bi, S Y Xiang et al. Determination of Phenolic Cpmpounds in Dendrobium chrysotoxum 
at Different Harvest Times [J]. Chinese Traditional and Herbal Drugs, 2005, 36(12):1885-1886

11. Q F Gong, H Zhou, X G Wang et al. Comparative Study on the Quality of Dendrobium officinale from Different Producing Areas [J]. Northern Horticulture, 2013(08) :162-165

12. W Q Xu, Y B Wang, Z R Sun. Antitumor Research Status Analysis of Dendrobium [J]. Chinese journal of Modern Applied Pharmacy, 2017, 34(1) :130-134

13. Y Y Zhang, Q Q Zhang, F H Wei et al. Rrogressive study of effects of erianin on antivancer activity[J]. Onco Targets and Therapy, 2019, 12 :5457-5465. 